\title{
Printed Multi-color Devices using Oxidative Electrochromic Materials
}

\author{
Daisuke Goto", Satoshi Yamamoto, Toshiya Sagisaka, Masato Shinoda, \\ Fuminari Kaneko, Keiichiro Yutani, Keigo Takauji, Yoshinori Okada, and \\ Tohru Yashiro
}

\author{
Ricoh Institute of Future Technology, Ricoh Co., Ltd. \\ 16-1 Shinei-cho, Tsuduki-ku, Yokohama, Kanagawa 224-0035, Japan \\ *daisuke.dg.gotoh@nts.ricoh.co.jp
}

\begin{abstract}
We developed new oxidative electrochromic materials based on triarylamines and benzidine. These were coated onto substrates and cured by UV irradiation to obtain polymer films. These materials emitted yellow, magenta and cyan colored light. In color devices using these materials, high transmittances of the uncolored states and high optical densities of the colored states were shown to be compatible. We demonstrated that CMYK and RGB can be expressed by mixing these materials. We also succeeded in prototyping EC decorative glass by combining these materials and using inkjet printing; this represents a new application for oxidative EC materials.
\end{abstract}

Keywords: Electrochromic devices, Inkjet printing, Multicolor patterning

\section{Introduction}

Electrochromism is a phenomenon in which electrochromic (EC) materials reversibly color and decolor because of electrochemical redox reactions [1]. Gadgets that utilize changes in the optical properties of EC materials are called "electrochromic devices" (ECDs). Many applications for ECDs have been proposed such as antiglare rear-view mirrors [2], sunglasses [3,4], eyewear $[3,4]$, and smart windows for airplanes and buildings [5-9].

Among the various kinds of EC materials that have been studied, and both inorganic and organic EC materials are known. Tungsten oxide [8] is known to be an inorganic EC material, while dialkyl viologens (1) are known to be reductive organic EC materials [10-12]. In addition, triphenylamine derivatives are known to be oxidative materials (Fig. 1) $[13-15]$.

We have synthesized cyan-, magenta-, and yellow-colored EC materials based on the viologen skeleton and demonstrated their use in full-color electronic paper [16-20].

Low drive voltage, high durability and high color density have been demonstrated using oxidation / reduction materials for both electrodes in ECDs. $[21,22]$. In addition, methods that mix two or more kinds of EC materials have been shown to provide intermediate colors $[23,24]$. To expand the range of applications, the development of oxidative materials, in addition to reductive materials, is considered important.

Well known oxidative EC materials include triphenylamine (2) and tetraphenylbenzidine (3) that are used in hole-transport layers of organic photoconductors (OPCs) and organic light emitting diodes (OLEDs) (Fig. 1).

It is known that triphenylamine emits cyan or blue light in its one-electron oxidation state, while tetraphenylbenzidine is an orange emitter [13-15]. However, these oxidative-type coloring materials have been unable to sufficiently reproduce yellow and magenta. Fine color control of yellow and magenta is a difficult objective.

We report the basic characteristics of EC devices that use these compounds, and their development to applied devices such as electrochromic filters and colored decorative glass. We also report the patterning process for cyan, magenta and yellow (CMY) oxidative EC materials. 
Reductive electrochromic materials

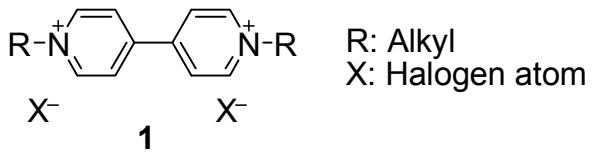

Oxidative electrochromic materials

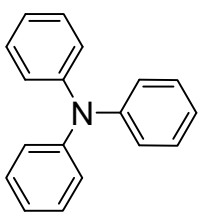

2

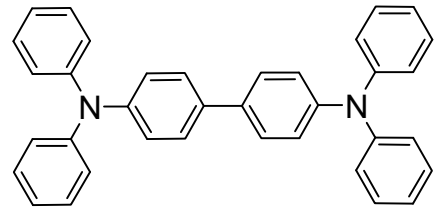

3
Fig. 1. Structure of reductive and oxidative electrochromic materials.

\section{Experimental}

2.1. Materials

In this study, we used CMY oxidative electrochromic materials based on triphenylamine modified with acryloyl groups for photo polymerization; these materials can be cured by UV irradiation.

\subsection{Electrochemical measurements}

Cyclic voltammetry experiments were performed using a normal potentiostat system (BAS Inc. ALS660B). Electrochemical measurements were performed in a three-electrode cell configured with a Pt-button $\left(0.02 \mathrm{~cm}^{2}\right)$ working electrode, a Pt-flag counter electrode, and a $\mathrm{Ag} / \mathrm{Ag}^{+}(10 \mathrm{mM}$ in tetrabutylammonium perchlorate (TBAP) $/ \mathrm{CH}_{2} \mathrm{Cl}_{2}$ ) reference electrode, which was calibrated to the ferrocene/ferrocenium $\left(\mathrm{Fc} / \mathrm{Fc}^{+}\right)$redox couple.

\subsection{Preparation of solutions of EC materials}

To the EC material $(140 \mathrm{mg})$ and poly(ethylene glycol) diacrylate (PEG400DA, $60 \mathrm{mg}$, Nippon Kayaku Co., Ltd.) in dry tetrahydrofuran (THF, 800 $\mathrm{mg}$ ) was added Irgacure ${ }^{\mathrm{TM}} 184$ (3 mg, BASF Co., Ltd.) to afford the required solution of the EC material.

\subsection{Fabrication of the ECDs}

Figure 2 depicts a schematic diagram of the EC devices fabricated in this study. Indium-tin-oxide (ITO) coated glass (Geomatec Co., Ltd.) was used as the substrate for the ECDs. The ITO glass was cut into $4.0 \times 4.0 \mathrm{~cm}$ squares, ultrasonically washed in IPA for $15 \mathrm{~min}$, and then dried under a flow of nitrogen. The ITO glass squares were then cleaned in UV/ozone for $15 \mathrm{~min}$. The previously prepared solutions of the EC materials (250 L) were spin-

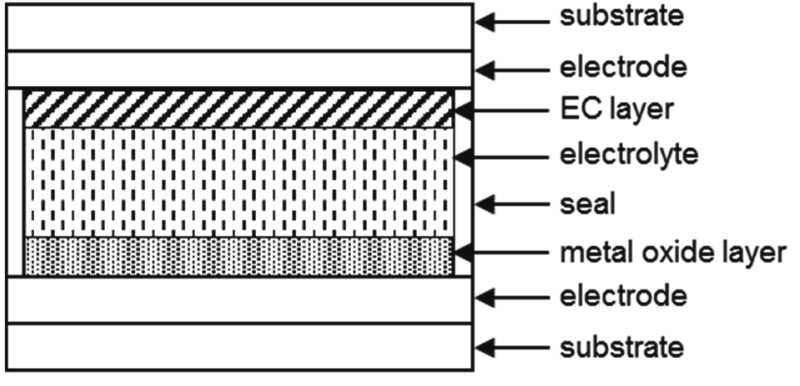

Fig. 2. Schematic image of the EC devices.

coated (1500 rpm, $30 \mathrm{~s})$ onto the ITO substrates and cured by UV irradiation $\left(0.6 \mathrm{~J} / \mathrm{cm}^{2}\right)$ using a high-pressure mercury lamp (USHIO Co. Ltd.) under a nitrogen atmosphere. Spacer particles (GS-270, Sekisui Chemical Co., Ltd.) were then coated onto ITO glass squares that were previously coated with $3.5-\mu \mathrm{m}$-thick tin oxide layers. Two ITO glass squares were sandwiched and the ECD gap was fixed at $80 \mu \mathrm{m}$ with spacer particles. The active area of each ITO glass device was controlled to be $9.0 \mathrm{~cm}^{2}(3.0 \times 3.0 \mathrm{~cm})$ using a UV-curable sealant (TB3035B, Three Bond Co., Ltd.). A $150 \mu$ L sample of an electrolyte containing 1-ethyl-3methylimidazolium bis(fluoro- sulfonyl)imide (EMIM-FSI, Kanto Chemical Co., Ltd), PEG400DA and Irgacure ${ }^{\mathrm{TM}} 184$, in a weight ratio of 60:38:2, was injected into two sandwiched ITO glass devices via pre-formed injection holes. The electrolyte was cured by UV irradiation under nitrogen and the injection holes were sealed with a UV-curable sealant. A voltage of $1.5 \mathrm{~V}$ was applied to both electrodes to color the ECD. The UV-vis spectrum was measured using an Ohtsuka Denshi Co., Ltd. LCD 5200 instrument.

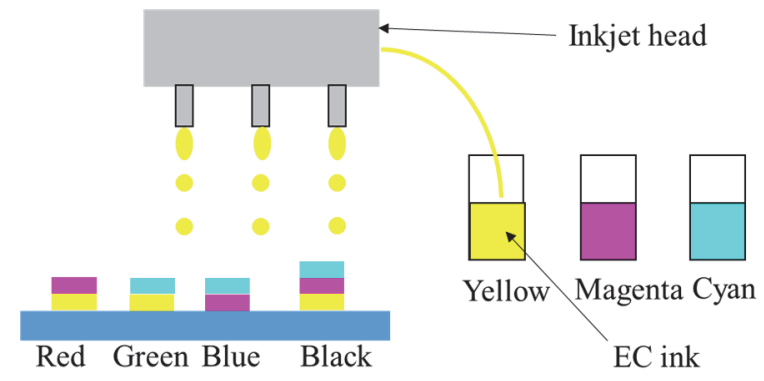

Fig. 3. Schematic image of inkjet printing using EC inks.

\subsection{Multilayer lamination by inkjet printing}

The inkjet (IJ) printing inks were prepared in the same manner as described above (see 2.3) except that the solvent used was cyclohexanone, instead of THF. Inkjet heads (Ricoh Co., Ltd. GEN4 (600 x 600 dpi)) were filled with the inks, devices fabricated by printing, as shown in Fig. 3, and cured by $\mathrm{UV}$ irradiation $\left(0.6 \mathrm{~J} / \mathrm{cm}^{2}\right)$. CMYK and RGB 
devices were fabricated by repeating this process up to three times with the appropriate sequence of inks. The contrasting color densities were expressed in a pseudo manner by applying the error diffusion method.

\section{Results and discussion}

3.1. Evaluation of optical and electrical properties

Figure 4 shows observed absorption spectra of the EC materials in devices. The observed absorption spectra were obtained when voltages of $1.5 \mathrm{~V}$ were applied to the various ECDs. They generally displayed CMY color.

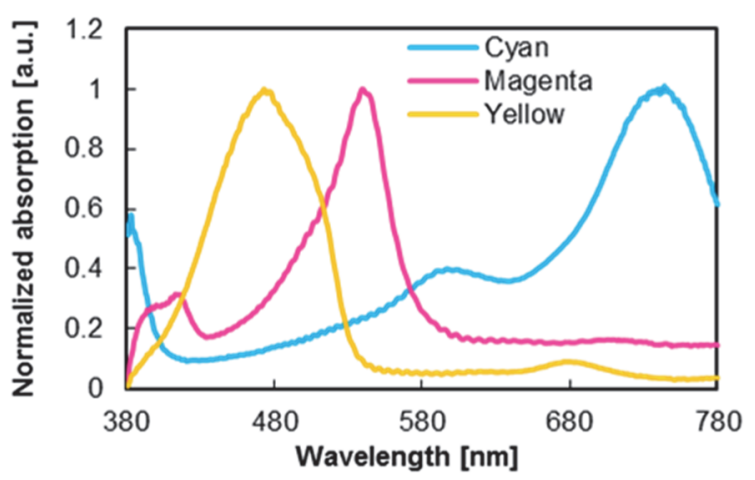

Fig. 4. UV-vis spectra of the EC materials.

(a)

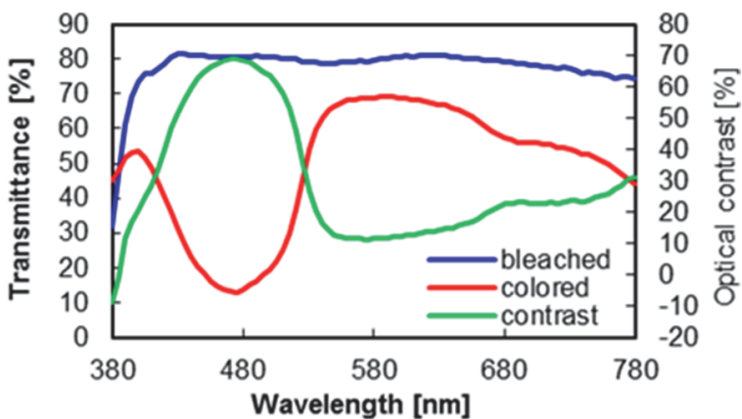

(b)

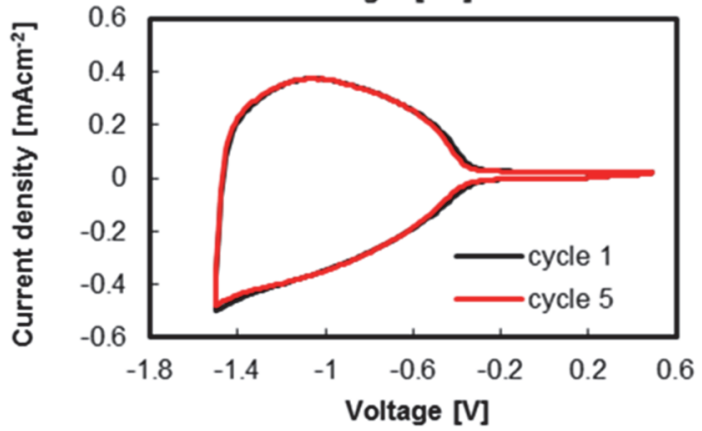

Fig. 5. Electrochemical characterization of the yellow EC device. a) Cyclic voltammogram (five cycles). b) Transmittance spectra of colored and decolored states (left axis) and corresponding optical contrast (right axis).
Figure 5 shows the results of the electrochemical characterization of the yellow EC device. The cyclic voltammogram (Fig. 5a) shows that the device is stable when driven over five cycles between -0.5 and $+1.5 \mathrm{~V}$. Fig. $5 \mathrm{~b}$ shows that transmittance exceeds $80 \%$ at $470 \mathrm{~nm}$ in the bleached state, and reaches almost $15 \%$ in the colored state; the corresponding contrast is about $70 \%$.

\subsection{Gradation control by inkjet printing}

Figure 6 shows images of contrasting color density in the yellow EC device that were expressed in a pseudo manner by applying the error-diffusion method. The electrochromic layer was composed of dots of about $60 \mu \mathrm{m}$ in diameter (Figs. $6 \mathrm{~b}$ and $6 \mathrm{c}$ ).

Figure 6 shows that eight gradations can be pseudo-expressed by differences in dot density.

(a)

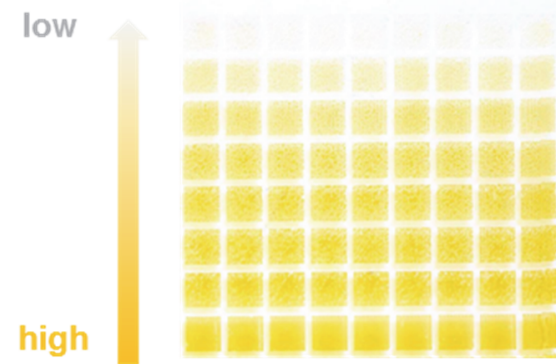

(b)
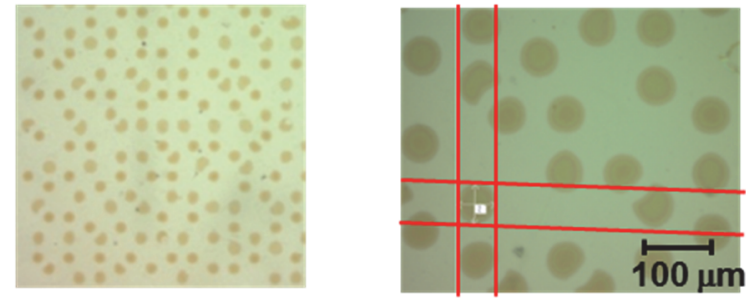

(c)
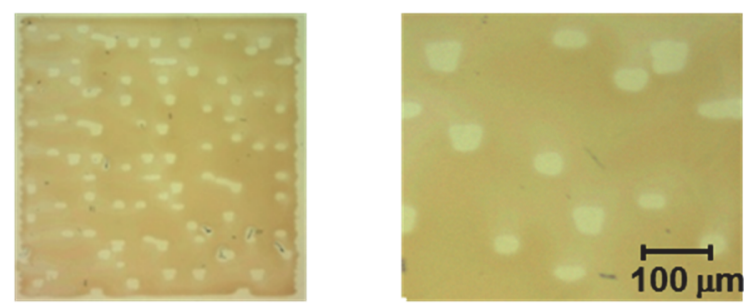

Fig. 6. Images depicting contrasting expression densities. a) Entire image. b) Microscope images of: (b) the lowcontrast density area (top line, Fig. 6a), and (c) the highcontrast density area (bottom line, Fig. 6a).

Figure 7 depicts changes in transmittance when a constant voltage is applied to ECDs in which mixtures of two dyes were laminated. The transmittance of each dye was constant (up to $600 \mathrm{~s}$ ) immediately after voltage application, and each dye was able to color independently without energy transfer. The maximum difference in the oxidation potentials of the CMY materials was $0.14 \mathrm{~V}$. Since the difference in the electrochemical reaction rates 
is relatively small, energy transfer between dyes is expected to be suppressed when they are mixed.

(a)

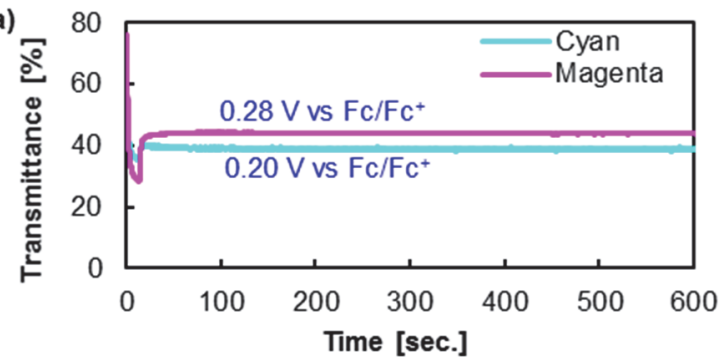

(b)

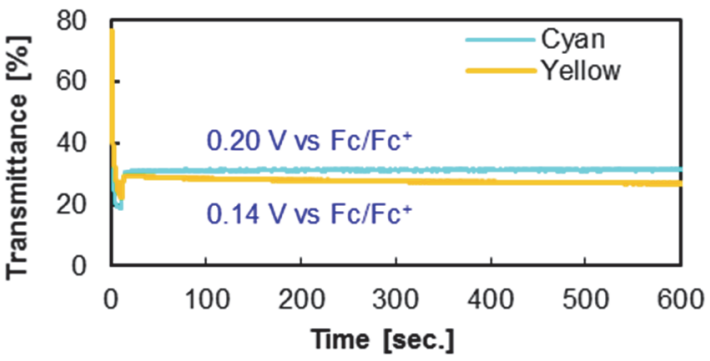

(c)

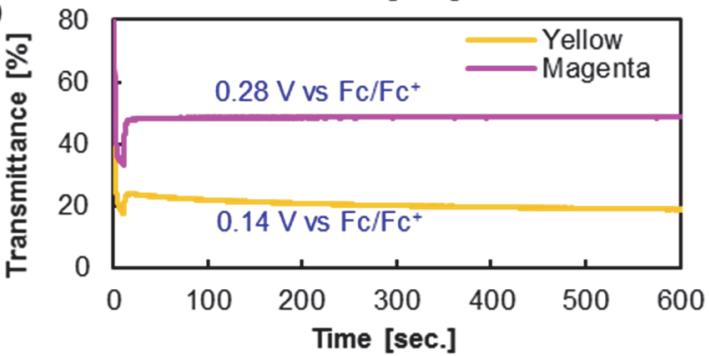

Fig. 7. Transmittance spectral transitions of EC devices composed of mixtures of two dyes. a) Cyan and magenta. b) Cyan and yellow. c) Yellow and magenta.

\subsection{Full color display by laminating CMY dyes}

Figure 8 depicts the CIE 1976 color space and photographs of the ECDs. By mixing two of the CMY dyes, it is possible to represent Red, Green and Blue (RGB), which are half tones. Black $(\mathrm{K})$ is also obtained by mixing three dyes. (a)

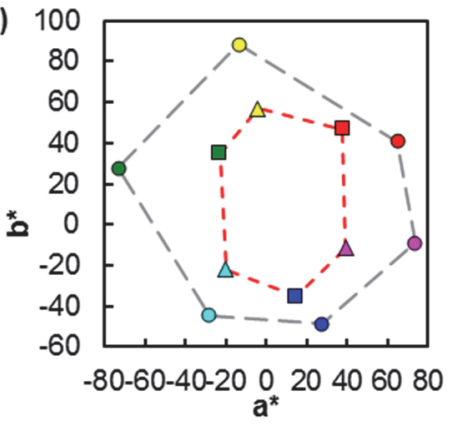

(b)

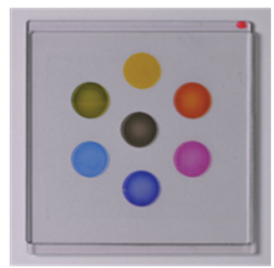

Fig. 8. CIE 1976 color space (left) and photograph (right) of the CMYK/RGB ECDs. The RGB color coordinates were estimated from the CMY coordinates.
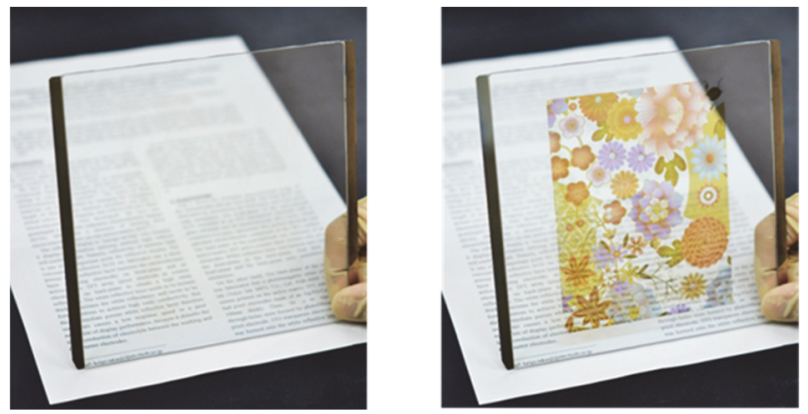

Fig. 9. Photographs of EC decorative glass (left: decolored, right: colored).

\subsection{EC application to colored decorative glass}

The CMY EC materials can be laminated and mixed. The all-solid-state devices exhibited constant memory properties; consequently, we succeeded in prototyping EC colored decorative glass using IJ printing. Figure 9 shows the decorated glass when decolored and colored. The transmittance in the decolored state was $80 \%$ or more, and conversion to the colored state occurred by applying a voltage of $1.5 \mathrm{~V}$ for 10 seconds; this state was retained even when the applied voltage ceased. In addition, by applying zero V for $15 \mathrm{~s}$, the glass returned to its initial state.

\section{Conclusions}

We demonstrated ECD device fabrication by IJ printing using UV curable CMY oxidative EC materials. In color devices using these materials, both high transmittances of the uncolored states and high optical densities of the colored states were shown to be compatible. We showed that CMYK and RGB could be expressed by mixing these materials. In the solid state, the color elements exhibit memory properties. We also succeeded in prototyping EC decorative glass by combining these materials and using IJ printing; this represents a new application for oxidative EC materials.

\section{Acknowledgement}

This work was supported in part by the New Energy and Industrial Technology Development Organization (NEDO) based of funds provided (2011-2015) by the Ministry of Economy, Trade and Industry, Japan (METI).

\section{References}

1. D. R. Rosseinsky and R. J. Mortimer, Adv. Mater., 13 (2001) 783.

2. J. H. Bechtel and H. J. Byker, U.S. Patent No. US4917477A, 1990.

3. A. M. Österholm, D. E. Shen, J. A. Kerszulis, R. H. Bulloch, M. Kuepfert, A. L. Dyer, and J. R. 
Reynolds, ACS Appl. Mater. Interfaces, 7 (2015) 1413.

4. K. Yutani, S. C. Kim, S. Yamamoto, Y. Okada, Y. Naijoh, S. Hirano, D. Gotoh, M. Inoue, T. Sagisaka, and T. Yashiro, Proc. IDW'13, (2013) 1311.

5. R. Baetens, B. P. Jelle, and A. Gustavsen, Sol. Energy Mater. Sol. Cells, 94 (2010) 87.

6. B. P. Jelle, A. Hynd, A. Gustavsen, D. Arasteh, H. Goudey, and R. Hart, Sol. Energy Mater. Sol. Cells, 96 (2012) 1.

7. B. P. Jelle, Sol. Energy Mater. Sol. Cells, 116 (2013) 291.

8. G. A. Niklasson and C. G. Granqvist, J. Mater. Chem., 17 (2007) 127.

9. R. J. Mortimer, Annu. Rev. Mater. Res., 41 (2011) 241.

10. I. Imae, Y. Takenaka, D. Tokita, Y. Ooyama, K. Komaguchi, and Y. Harima, Chem. Lett., 37 (2008) 964.

11. H. C. Moon, T. P. Lodge, and C. D. Frisbie, Chem. Mater., 27 (2015) 1420.

12. J. Deng, X. Fu, G. Wang, L. Wu, and J. Huang, Electrochimica Acta, 85 (2012) 195.

13. Y. Chuang, H. Yen, J. Wu, and G. Liou, $A C S$ Appl. Mater. Interfaces, 6 (2014) 3594.

14. P. J. Low, M. A. J. Paterson, A. E. Goeta, D. S. Yufit, J. A. K. Howard, J. C. Cherryman, D. R. Tackley, and B. Brown, J. Mater. Chem., 14 (2004) 2516.

15. H. Yen, K. Lin, and G. Liou, J. Mater. Chem., 21
(2011) 6230.

16. T. Yashiro, S. Hirano, Y. Naijoh, Y. Okada, K. Tsuji, M. Abe, A. Murakami, H. Takahashi, K. Fujimura, and H. Kondoh, SID 11 Digest, (2011) 42.

17. Y. Naijoh, T.Yashiro, S. Hirano, Y. Okada, S. Kim, K. Tsuji, H. Takahashi, K. Fujimura, and H. Kondoh, Proc. IDW'11, (2011) 375.

18. Y. Okada, T. Yashiro, Y. Naijoh, S. Hirano, S. Kim, K. Tsuji, H. Takahashi, K. Fujimura, and H. Kondoh, Proc. IDW'12, (2012) 641.

19. T. Yashiro, Y. Okada, Y. Naijoh, S. Hirano, T. Sagisaka, D. Gotoh, M. Inoue, S. Kim, K. Tsuji, H. Takahashi, and K. Fujimura, Proc. IDW'13, (2013) 1300.

20. K. Takauji, Y. Okada, S. Yamamoto, D. Gotoh, M. Shinoda, T. Sagisaka, Y. Matsuoka, F. Kaneko, and T. Yashiro, Proc. ICAI 2015, (2015) 135.

21. M. Li, Y.Wei, J. Zheng, D. Zhu, and C. Xu, Org. Electron., 15 (2014) 428.

22. D. Cummins, G. Boschloo, M. Ryan, D. Corr, S. N. Rao, and D. Fitzmaurice, J. Phys. Chem. B, 104 (2000) 11449.

23. R. H. Bulloch, J. A. Kerszulis, A. L. Dyer, and J. R. Reynolds, ACS Appl. Mater. Interfaces, 7 (2015) 1406.

24. B. Chen, S. Kao, C. Hu, M. Higuchi, K. Ho, and Y. Liao, ACS Appl. Mater. Interfaces, 7 (2015) 25069. 